\title{
Azathioprin - weiterhin indiziert beim Morbus Crohn
}

Studienergebnisse wie auch Leitlinien geben nur bedingt Hinweise auf das optimale Management bei chronisch entzündlichen Darmerkrankungen (CED). Denn es handelt sich bei Morbus Crohn und Colitis ulcerosa um komplexe Krankheitsbilder, bei denen oft Einzelfallentscheidungen gefragt sind. Welcher Stellenwert dabei Azathioprin zum Remissionserhalt bei Morbus Crohn zukommt, wird derzeit kontrovers diskutiert.

Für nicht ausreichend belegt hält Prof. Dr. Christian Maaser, Lüneburg, dass durch eine Therapie mit dem Immunsuppressivum bei Crohn-Patienten ein OP-sparender Effekt erzielt werde, erklärte der Gastroenterologe beim Falk Seminar „CED kontrovers“ in Hamburg. Er merkte außerdem kritisch an, dass Hinweisen zufolge bei einer langfristigen Behandlung mit Azathioprin das Malignomrisiko steigen könne. Gegen einen unkritischen Einsatz des Immunsuppressivums wandte sich auch Privatdozent Dr. Jürgen Büning, Lübeck. Er er- klärte aber, dass Azathioprin (z.B. Azafalk $^{\circledR}$ ) dennoch weiterhin einen festen Stellenwert bei der Erhaltungstherapie des steroidabhängigen Morbus Crohn besitzt, um die Steroiddosierung schrittweise reduzieren zu können.

\section{Therapieoptimierung durch Spiegelkontrolle}

Zeigt sich unter Azathioprin nicht der erwartete Therapieerfolg, sollte kontrolliert werden, ob tatsächlich im individuellen Fall ausreichend hohe Wirkstoffspiegel erreicht werden. $\mathrm{Zu}$ prüfen ist dies durch eine 6-Thioguanin-Nukleotid-Spiegelkontrolle (6-TGN). Diese ist nach etwa vier Wochen indiziert und zeigt, ob das Immunsuppressivum ausreichend hoch dosiert ist, was eine Voraussetzung für die Krankheitskontrolle darstellt. Gegebenenfalls muss die Dosierung angepasst werden.

Limitierend können hierbei Nebenwirkungen sein, wie Büning darlegte. In einem solchen Fall rät er dazu, die Azathioprin-Dosis auf zwei Einnahmezeitpunkte zu teilen, die Therapie auf 6-Methyl-Mercaptopurin (6-MMP) als wirksamen Metaboliten von Azathioprin umzustellen oder zusätzlich zu Azathioprin Allopurinol zu verordnen. Die Möglichkeit einer zusätzlichen Gabe von Allopurinol, das eine Erhöhung der Bioverfügbarkeit von Azathioprin bewirkt, wird jedoch noch zu selten genutzt, so Büning.

(Christine Vetter)

\section{Einsatz von Biologika: Original oder Biosimilar?}

Sind Biosimilars den Originalpräparaten in ihrer Wirksamkeit ebenbürtig und sicher? Für den forcierten Einsatz von Biosimilars, wie sie zum Beispiel bei Infliximab bereits verfügbar sind, spricht nach Professor Dr. Andreas Sturm, Berlin, vor allem der günstigere Preis der Präparate. $\mathrm{Zu}$ bedenken aber ist, dass die Medikamente mit Hilfe lebender Mikroorganis- men hergestellt werden, so dass stets eine gewisse Variabilität gegeben ist.

\section{Einsatz bei Arthritis und CED}

Als problematisch bewertet es zudem Privatdozent Dr. Andrea Pace, Neumünster, dass die Zulassung der Biosimilars auf der Basis einer klinischen Studie in nur einem Indikationsbereich erfolgt und die

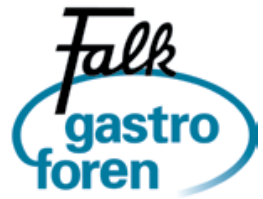

Besuchen Sie das nächsteFalkGastroForum „Hepato-/Gastroenterologie zwischen Wissenschaft und Praxis - Was bleibt - Was kommt?" am Samstag, den 5. November 2016 in Aachen; Infos: www.drfalkpharma.de/veranstaltungen
Daten dann auf andere Indikationen extrapoliert werden. So wurde das Biosimilar zu Infliximab lediglich bei Patienten mit rheumatoider Arthritis untersucht, kann aber auch bei Patienten mit CED angewandt werden. „Es gibt bislang kein biosimilares Präparat, das bei Patienten mit Morbus Crohn oder Colitis ulcerosa geprüft wurde“, so Pace. „Wir müssen uns somit darauf verlassen, dass die bei der rheumatoiden Arthritis ermittelten Ergebnisse auch bei Patienten mit chronisch entzündlicher Darmerkrankung zutreffen“.

(Christine Vetter)

Falk Seminar "CED kontrovers", Hamburg, 11. Juni 2016, Veranstalter: Falk Foundation e.V. 